\title{
A Self-Adaptive Method of Multi-scale POI Visualization in Indoor Map
}

\author{
Yi Xiao ${ }^{\text {a }}$, Tinghua $\mathrm{Ai}^{\mathrm{a}}{ }^{*}$, Mengying Jiang ${ }^{\text {a }}$, Chen Weng ${ }^{\text {a }}$, Miqi Wang ${ }^{\text {a }}$ \\ ${ }^{a}$ Wuhan UniversityYiXiao,yixiao_whu@outlook.com,Tinghua Ai,tinghuaai@whu.edu.cn, Mengying Jiang,Dameng_Jmy@163.c \\ om, Chen Weng,2018282050182@whu.edu.cn, Miqi Wang,2018202050074@whu.edu.cn \\ * Corresponding author
}

Keywords: POI data, Adaptive visualization, Indoor map, Multi-scale

\begin{abstract}
:
As an important representation of LBS (Location Based Service), the indoor map provides users with facility location, indoor navigation, wayfinding and other necessary services. Compared with the common map, the indoor map has characteristics of detailed representation, 3D visualization, and real-time reaction. As far as POI (Point of Interest) representation is concerned, the common urban map just visualizes the location and the basic attribute in a simple way by relatively large scale representation. In the indoor map, however, the POI needs to represent the 3D location (level, longitude, latitude), the access path, and some detailed service information. Take the map of shopping mall as an example, traditionally, some services visualize restaurants that should have been distributed at different layers in a crowding way, with icons touching and overlapping with each other. Therefore, the LOD (level of detail) strategy needs to be applied to obtain adaptive representation for users with different objectives.
\end{abstract}

To satisfy the requirements of the new indoor map about POI visualization, this study explores the multi-scale method in 3D indoor mapping. We develop an indoor map which is integrated into an outdoor base map and all the floors of the whole building are stacked vertically. In this way, the problem of icon clustering could be overcome to an extent, POI data are visualized vertically in indoor space, each floor can be taken out like opening a drawer in order to make sure that all the POIs can be seen clearly. We adopt a scale adaptive strategy to visualize POI data in the indoor map. At large scale, the general floor distribution indicating the main function of each floor and functional partition of each floor is represented by different colors. At medium scale, POI data are visualized by simple symbols which can represent basic attribute information. At small scale, detailed service information (e.g., opening hours, number of seats remaining, price range and user ratings of a restaurant) of a POI that users are particularly interested in is described by well-designed map symbols based on the combination of visual variables. In addition, our POI visualization method is user-adaptive. Different data, symbols or color schemes will be shown for users with different age group, gender or purpose of using map. Because this indoor map is adaptive to both scale and user, each scale of the map can provide different map users with decision support from a different perspective.

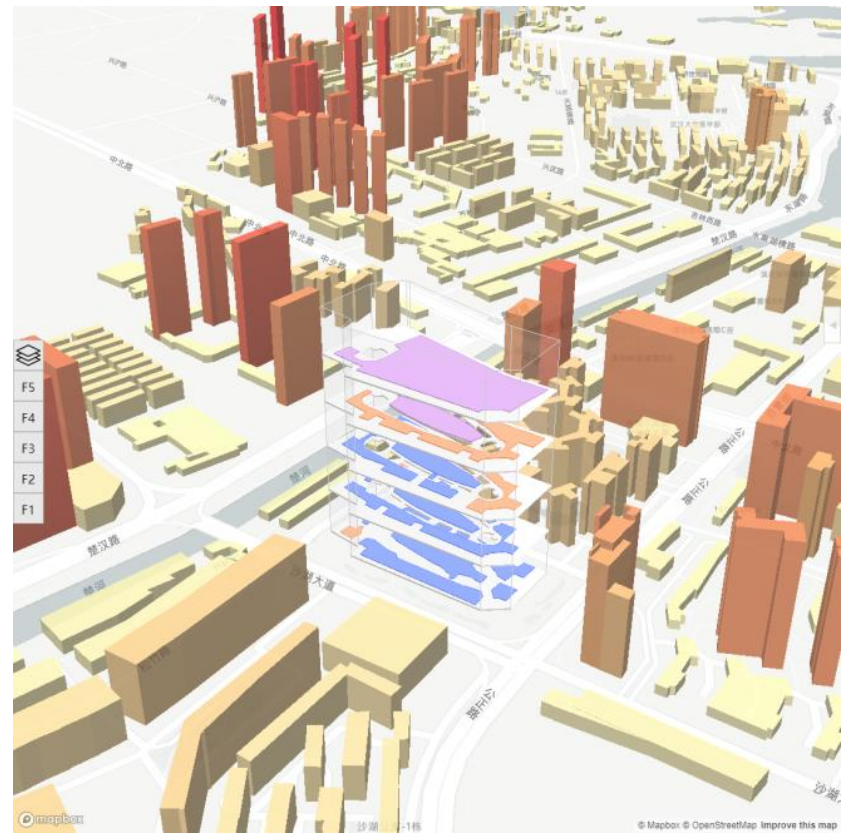

(a)

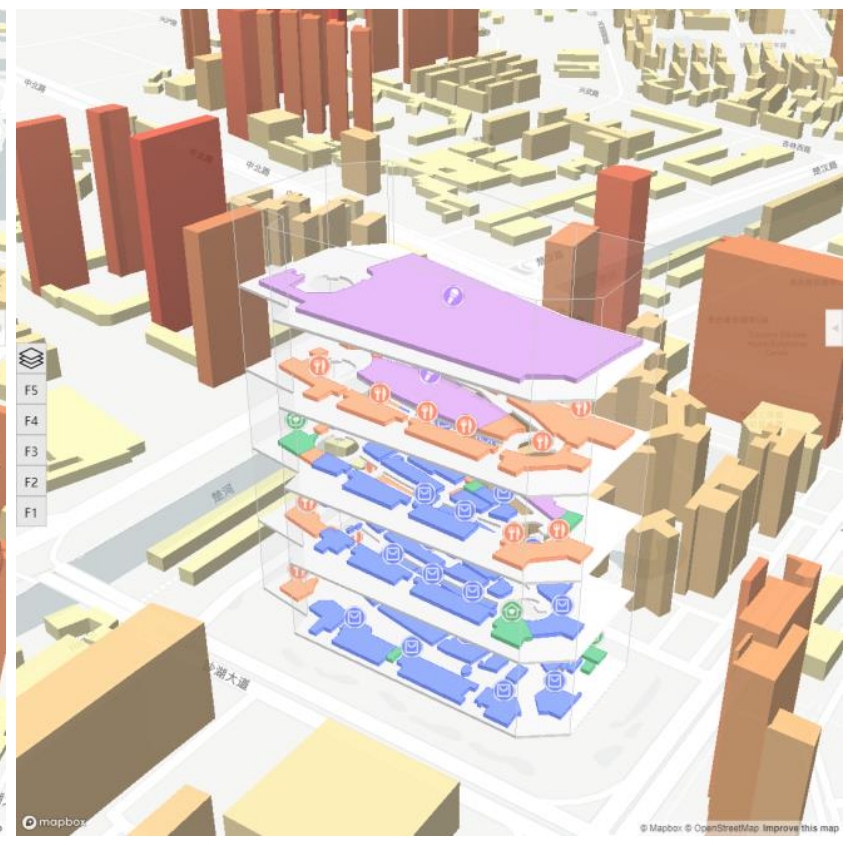

(b) 


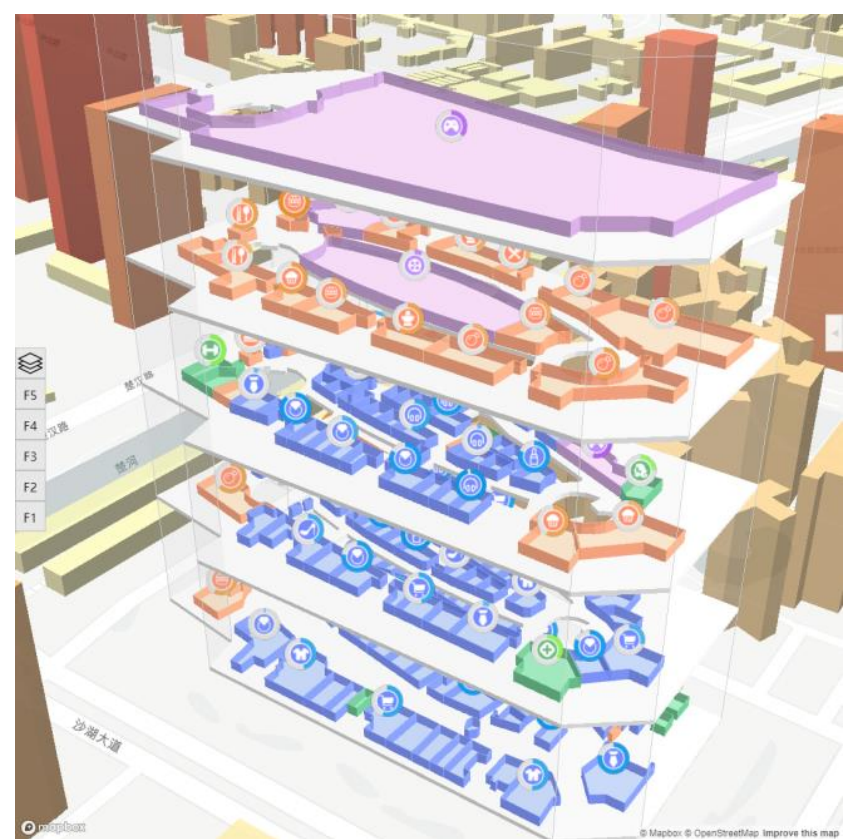

(c)

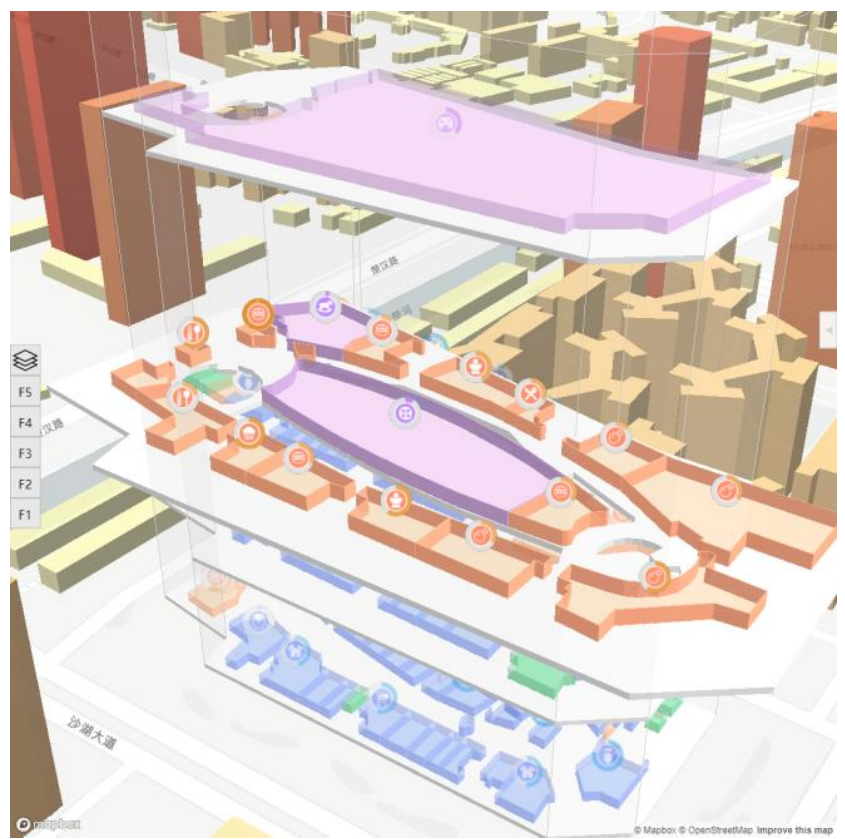

(d)

Figure 1. Multi-scale indoor map. (a) Large scale: General function division of each floor (b) Medium scale: POI data are visualized by simple symbols (c) Small scale: detailed room distribution and detailed symbols for POI data (d): Visual effect like opening a drawer 